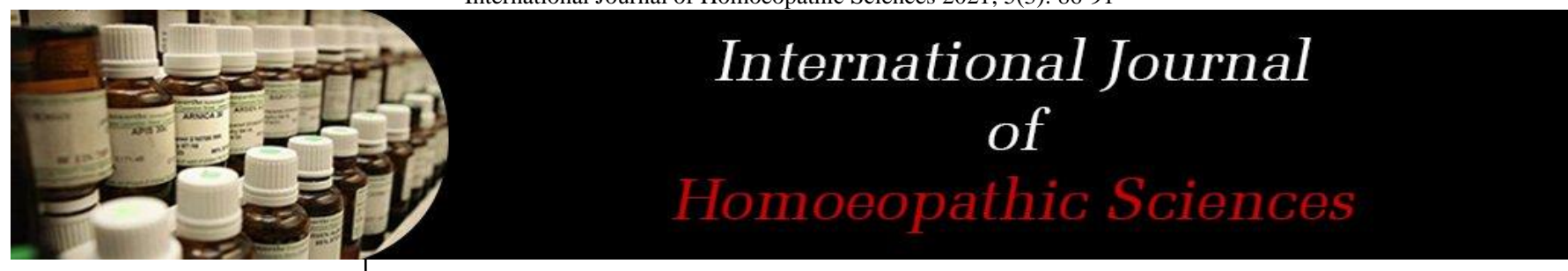

E-ISSN: 2616-4493 P-ISSN: 2616-4485 www.homoeopathicjournal.com IJHS 2021; 5(3): 86-91

Received: 22-05-2021

Accepted: 24-06-2021

Dr. Siddabathina Uma Devi Department of Homoeopathic Materia Medica, Guru Mishri Homoeo Medical College, Maharashtra, India
Corresponding Author: Dr. Siddabathina Uma Devi Department of Homoeopathic Materia Medica, Guru Mishri Homoeo Medical College, Maharashtra, India

\title{
Abnormal uterine bleeding and its homoeopathic approach
}

\section{Dr. Siddabathina Uma Devi}

DOI: https://doi.org/10.33545/26164485.2021.v5.i3b.411

\begin{abstract}
Many women have variations in their menstrual cycles such as changes in frequency, duration, amount of flow or spotting between the periods. This Abnormal Uterine Bleeding is common debilitating condition in women. It can occur between Menarche to Menopause in Ovulatory or Anovulatory cycles. Many structural causes like polyps, fibroids; functional and coagulopathies can lead to abnormal uterine bleeding. Excessive Menstrual Bleeding has several adverse effects including Anaemia, Iron deficiency and reduced quality of life. It affects medical and social health of women. Medical management is the preferred initial approach for hemo-dynamically stable patients to avoid surgical risks and preserve fertility. Knowledge of nature of individual sickness is necessary to prescribe and this depends upon study of symptoms prior to the pathology. Hahnemannian Homeopathy supplied a law to knit together the two branches of medical knowledge and has thereby put the art of medicine on a scientific basis.
\end{abstract}

Keywords: abnormal uterine bleeding, uterine haemorrhage, AUB, uterine bleeding

\section{Introduction}

Abnormal uterine bleeding is most common gynaecological symptom that describes irregularities in the menstrual cycle involving frequency, regularity, duration, and volume of flow outside of pregnancy. Up to one-third of women will experience abnormal uterine bleeding in their life, with or without major clinical conditions, most commonly occurring at menarche and peri-menopause. The normal menstruation has a class interval of 21-35 days, flow lasting 4-5 days with 20 to 80 milliliters of blood loss ${ }^{[1]}$. An average woman can expect roughly 400 Menstrual Cycles during her reproductive life span and it is estimated that up to $20 \%$ of women are suffering from excessive blood loss ${ }^{[19]}$. Excessive Menstrual Bleeding has several adverse effects including Anaemia, Iron deficiency and reduced quality of life. It affects medical and social health of women.

As per great saying of Dr. Allen, A woman should have no suffering at the menstrual period; there should be but a simple consciousness of its presence, a little lagging of the forces of life, a pause, as it were, for the preparation indeed, we should not have more than the simple consciousness of the presence and action of any organ, yet we find in the menstrual function of women, every degree of suffering even to the anguish of death, as the organs attempt to perform their periodical work. The sexual organs of the women of today are the great centers of disease ${ }^{[20]}$. To conserve the fertility and avoid potential complications, Homoeopathy serves as a key role in the treatment of Abnormal Uterine Bleeding as the first line of Medical management. Apart from conventional methods of treatment, Homoeopathy can rectify the dynamic changes from interior as a whole constitutionally.

\section{Prevalence}

According to a Doctor Centric Survey conducted in 2019, prevalence reported is $32.72 \%$ of females visit their clinic due to Abnormal Uterine Bleeding (AUB) ${ }^{[5]}$. Many studies are limited to Heavy menstrual bleeding, when comes to inter-menstrual bleeding or irregular menses, incidence rises to $35 \%$ or greater ${ }^{[10]}$. Another recent research study concludes that the incidence ranges about $22.02 \%$ and more common in the age group of 31-40 years ${ }^{[6]}$. Risk of malignant ovarian tumors increases with age. A US population based survey of ages 18-50 reported an annual prevalence rate of AUB as 53 per 1000 women ${ }^{[27]}$. 


\section{Types}

Abnormal uterine bleeding can also be divided into acute versus chronic. Acute AUB is the excessive bleeding that requires an immediate intervention to prevent additional blood loss. Acute AUB can occur on its own or superimposed on chronic AUB, which refers to irregularities in menstrual bleeding for most of the previous 6 months ${ }^{[21]}$.

\section{Etiology}

The causes of abnormal uterine bleeding vary by age; anovulatory bleeding seen commonly in adolescents and perimenopausal women. The most frequent cause of irregular bleeding is hormonal while pregnancy related causes (spontaneous abortion, ectopic pregnancy) also be considered. A variety of terms are used to describe abnormal menses-Menorrhagia, Metrorrhagia, Menometrorrhagia, Hypermenorrhoea, Hypomenorrhoea, Oligomenorrhoea, Amenorrhoea which are resulted from different etiological factors ${ }^{[4]}$.

Causes like Spontaneous abortion, Exogenous Hormones, Endocrine Causes like hyperthyroidism, hypothyroidism, Diabetes Mellitus, anatomic causes like Leiomyomas, Endometrial Polyps, Endocervical Polyps, Cervical lesions (Condylomata), Infectious causes like Cervicitis, Pelvic inflammatory disease, herpes simplex virus ulcerations; Neoplasia, Endometriosis, Adenomyosis and other Coagulopathies lead to Abnormal uterine bleeding.

According to PALM-COEIN classification, International Federation of Obstetrics and Gynecology (FIGO) classify the underlying etiologies of abnormal uterine bleeding. The first portion, PALM, describes structural issues. The second portion, COEI, describes non-structural issues ${ }^{[9]}$. The classification is as follows:

- P: Polyp

- A: Adenomyosis

- L: Leiomyoma

- M: Malignancy and hyperplasia

- C: Coagulopathy

- O: Ovulatory dysfunction

- E: Endometrial disorders

- I: Iatrogenic

- N: Not otherwise classified

\# An observational study regarding causes of AUB shown highest incidence of Leiomyoma among all other etiological factors of PALM-COEIN classification ${ }^{[8]}$.

\section{Patho-Physiology}

The pathophysiology of abnormal uterine bleeding (AUB) is as varied as the classification of the disease. AUB can occur from pelvic pathology such as a distortion of the endometrial cavity due to fibroids or endometrial protrusions into the cervix or vagina (polyps), or because of friable endometrial tissue. The friable endometrial tissue is probably caused by unopposed estrogen which causes it to become friable, vascular, and lacking sufficient stromal support which associate to heavy and continuous uterine bleeding.

The organs of the female reproductive system include ovaries (the female gonads); the uterine or fallopian tubes, or oviducts; the uterus; the vagina; and external organs ${ }^{[2]}$. Female reproductive functions can be divided into two major phases- The Menstrual cycle (Preparation of female body for conception \& pregnancy) and The Period of Pregnancy ${ }^{[3]}$. The normal human menstrual cycle can be divided into two segments: the ovarian cycle and uterine cycle, based on the organ under examination.

The ovarian cycle can be further divided into follicular and luteal phases where as the uterine cycle is divided into proliferative and secretory phases ${ }^{[4]}$. A 28 day cycle is divided into:

- The initial 14 days where the follicle grows is called the proliferative or follicular phase.

- The remaining 14 days after the follicle has ruptured [ovulation] and released the ovum [egg] is called the secretory or luteal phase.

The proliferative phase is characterized by a predominance of oestrogen over progesterone and upsurge of endometrium. Progestereone production is triggered by ovulation; there the secretory phase begins. The secretory phase is noticeable with a reaction to the combination of oestrogen and progesterone and stabilization in the thickness of the endometrium. Menstrual bleeding occurs after secretion of oestrogen and progesterone tapers off. During menses, thrombin plugs restrain blood loss, later on, vasoconstriction of the spiral arterioles is responsible for haemostasis. When ovulation does not take place, progesterone levels do not rise; thereby typical cyclic withdrawal of oestrogen and progesterone cannot occur. Normal menstrual cycles are characterized by a cycle length of 28 days, duration of flow of 4 days, and a blood loss of $40 \mathrm{ml}$. Menstruation is regulated by Hypothalamuspituitary-ovarian cycle. Developing follicles secrete oestrogen to stimulate thickening of Endometrium.

During an ANOVULATORY cycle, there is no formation of the corpus luteum. Thus, there will be no normal cyclical secretion of progesterone and oestrogen stimulates the endometrium unopposed. The endometrium continues to proliferate in the absence of progesterone, it eventually outgrowing its blood supply then sloughs incompletely and bleeds irregularly and sometimes profusely or for a long time. When this abnormal process takes place repeatedly, the endometrium can become hyperplastic, sometimes with atypical or cancerous cells. Anovulatory AUB can be resulted from any disorder or condition that causes anovulation. Anovulatory AUB occurs at unpredictable times and in irregular patterns and is not accompanied by cyclic changes in basal body temperature.

In OVULATORY abnormal uterine bleeding, secretion of progesterone is prolonged; resulting in irregular shedding of the endometrium, probably due to low estrogen levels near the threshold for bleeding (as it occurs during menses). Ovulatory AUB can occur if estrogen levels are high, resulting in amenorrhea alternating with irregular or prolonged bleeding which is commonly seen in obese women. Ovulatory AUB tends to occur excessive bleeding during regular menstrual cycles. Women may possibly have other symptoms of ovulation, such as premenstrual symptoms, breast tenderness, mid-cycle cramping pain (mittelschmerz), a change in basal body temperature after ovulation and sometimes dysmenorrhea.

\section{Anovulation is most often seen}

- Secondary to Polycystic Ovarian Syndrome.

- Idiopathic (occurring when gonadotropin levels are normal)

- Sometimes anovulation results from Hypothyroidism. 
During perimenopause, anovulatory AUB may suggest as an early sign of ovarian insufficiency or failure; follicles are still developing but, despite increasing levels of folliclestimulating hormone (FSH), do not produce enough estrogen to trigger ovulation. About $20 \%$ of women have Endometriosis that presents anovulatory AUB due to unknown mechanisms. Chronic anovulation may lead to irregular bleeding, prolonged unopposed estrogen stimulation of the endometrium, and increased risk of endometrial cancer.

\section{Ovulatory AUB may occur in}

- Polycystic ovary syndrome (as progesterone secretion is prolonged)

- Endometriosis, which does not affect ovulation

Other causes of Ovulatory AUB are short follicular phase and luteal phase dysfunction (due to inadequate progesterone stimulation of the endometrium); a rapid decrease in estrogen before ovulation can cause spotting.

\section{Diagnosis}

Assessing blood loss in a patient becomes difficult as their perception of menses gets deviated. A detailed history taking, careful planning of laboratory evaluation and specific management approaches is required. A delay in diagnosing bleeding disorder interferes with woman's physical, emotional, social and material quality of life.

After detailed physical examination and ordering blood tests, a doctor may recommend an imaging study in order to evaluate the appearance of the uterus, including the shape, size, and presence of any obvious structural abnormality. An array of techniques is available to visualize the uterus and pelvic organs.

Ultrasound (Sonogram): USG is a procedure which uses high-frequency sound waves to produce a picture of the pelvic structures. It is the most commonly used imaging method for the pelvic organs without involving the exposure of radiation. The ultrasound is frequently performed on the surface of abdomen and pelvis as well as from within the vagina.

Endometrial Abnormalities may also be detected by a Hysterosalpingogram (HSG). This involves the slow injection of an iodine-containing solution into the uterine cavity under $\mathrm{x}$-ray guidance so that the contours of the endometrium and fallopian tubes can be seen.

Unusually, computerized tomography (CT) and magnetic resonance imaging (MRI) can be used to depict a threedimensional image of internal organs including the uterus. MRI is often helpful than CT in visualizing pelvic structures and particularly useful in patients where adenomyosis is suspected.

\section{Treatment}

The individual therapy aims at reducing specific cause of abnormal uterine bleeding. Structural abnormalities of the reproductive tract such as fibroids, polyps, or scar tissue can usually be treated during hysteroscopy. Surgical intervention through hysteroscope removes or corrects structural abnormalities within the uterine cavity. Generally, patients can get back to their normal activities within 24 hours after hysteroscopy. Serious complications are very rare.

Women who have adequate levels of oestrogen with no ovulation can be treated effectively with synthetic progestins such as medroxyprogesterone acetate. Other progestins, including natural progesterone, are available as oral capsules, vaginal suppositories, or intramuscular injections and also effective in promoting complete shedding of the endometrial lining. Many patients can be treated with low-dose combination oral contraceptives (OCs), which provide both oestrogen and progestins and promote regular menstruation. This may be useful choice for individuals who also desire birth control.

Menorrhagia: For women with menorrhagia (excessively prolonged or heavy menstruation), the administration of an oestrogen may be recommended to arrest the bleeding temporarily and stabilize the endometrial lining. Often an endometrial biopsy is recommended under such circumstances. Heavy uterine bleeding may be controlled with the use of low-dose Oral Contraceptives (OC).

Tranexamic acid is useful for episodes of heavy bleeding. This orally administered medication have shown to cause a major reduction in menstrual blood flow. Levonorgestrelcontaining IUDs are useful in treating DUB and may be the most effective therapy. Prostaglandin synthesis inhibitors, including ibuprofen and related compounds also showed reduction of excessive menstrual bleeding in some women.

Surgical intervention not only includes Hysterectomy but also include removal of any uterine abnormalities with the use of the hysteroscope. Surgical removal of fibroids may be recommended for women with menorrhagia that didn't respond to hormonal therapy. The type of surgical technique depends upon both the size and location of the fibroids. Myomectomy may be performed through hysteroscopy, laparoscopy or laparotomy (with abdominal incision). Hysterectomy also can be done through several different routes i.e., vaginal, laparoscopy, laparotomy.

Endometrial ablation can be recommended for a woman who has no desire for future fertility. This procedure, which is performed under general anesthesia, uses thermal radiofrequency, cryosurgery (freezing), or a laser beam for the removal of endometrial lining and generally corrects heavy menstrual flow. Prior to the procedure to be done, it is important to exclude serious endometrial lesions. Though the goal is to stop menstrual bleeding completely, most often menstrual periods become lighter following this procedure. Ablation should not be performed in women desiring future fertility. Only in compulsive conditions, hysterectomy can be recommended for heavy menstrual bleeding.

Pituitary and Thyroid Glandular Dysfunction Disorders can cause anovulation and menstrual irregularity. Individuals with hypothyroidism can be treated with daily oral intake of thyroid hormone medication. Hyperthyroidism can be treated with oral medications, radioactive iodine, or surgical excision of all or part of the thyroid gland. The treatment of hyperprolactinemia is generally treated with the oral medication cabergoline or bromocriptine. Individuals with elevated levels of prolactin may be advised to have an imaging study, such as a CT scan or MRI, of the pituitary gland to determine the presence of pituitary lesion. Surgical excision of pituitary adenomas is done when the tumour is large or involving vital organs.

Regular exercise and/or change in dietary habits (to reduced calorie intake or a vegetarian diet) can help in hormonal 
balance and that regulates irregular bleeding. An elevated FSH level may indicate impending ovarian failure, either due to menopause or to early menopause that is known as premature ovarian failure (POF).

Polycystic Ovary Syndrome (PCOS): Treatment of PCOS depending upon the goal whether it is of restoration of fertility or regular menstruation or both. Intermittent progestin therapy to attain fertility; oral contraceptives may be recommended to reestablish regular bleeding episodes and to reduce the risk of hyperplasia and cancer. Oestrogen and progestin together, as in OCs, may be more effective than progestin therapy. PCOS can be associated with high blood pressure, heart disease, acne, excess body hair (hirsutism), obesity, and diabetes mellitus, so proper medical attention and treatment are important.

\section{Homoeopathic View}

As mentioned by Dr. Cowperthwaite, Abnormal uterine functioning is but a symptom of various existing pathological states of the generative organs. It has been always arises from grave constitutional diseases. It is evident, therefore, that the condition itself can have no established pathology, nor is it probable that menstrual abnormality ever gives rise to pathological states, it being itself a result rather than a cause of the many constitutional diseases with which it is associated and the existence of which has been erroneously attributed to the failure of the menstrual function ${ }^{[13]}$.

Dr. Kent says...Homoeopathy is women's best friend. It respects her modesty, reserves her womanhood, relieves her of the many ailments peculiar to her sex $\&$ habits and does it all more pleasantly ${ }^{[14]}$.

The approach to the study of the case and the approach to the study of the Materia Medica are essentially the same-the Materia Medica is the facsimile of the sickness ${ }^{[15]}$.

Homoeopathy has wide range of remedies for abnormal uterine bleeding depending upon the causative factors and totality of symptoms. Some of the remedies listed below:

Actea Racemosa: A sensation of weight and bearing down in the uterine region with a feeling of weight and torpor in the lower extremities. Cramps and tenderness in the hypogastric region. Profuse flow of a passive character, the blood being dark and coagulated, attended with great nervous and mental irritability. Dysmenorrhea occurring in rheumatic women. Menstrual colic from retroversion [16]. Threatened abortion in rheumatic or hysterical women, with leucorrhoea.

Aletris Farinosa: Pressure in the region of the uterus. Habitual tendency to abortion. Prolapsus uteri from muscular atony. Profuse discharge of dark color in consequence of con gestion of the uterus or ovaries. Menstrual discharge dark and coagulated.

Janosia Ashoka: Various uterine disorders, astringent menstrual troubles chiefly menorrhagia. Menstrual discharge is scanty, pale, watery, foul smelling, blackish. Amenorrhoea at puberty with headache. Hysteria with loss of appetite [17]. Extensively employed in uterine ailments especially menorrhagia with fibroids; leucorrhoea with delayed menses. Headache due to suppressed menses ${ }^{[18]}$.

Lilium Tigrinum: Manifests powerful influence over pelvic organs and adapted to many reflex states dependent on some pathological condition of uterus and ovaries. Congestion of uterus. Ovarian pains with palpitations, mental symptoms. Menses cease while resting. Menses early, scanty, dark, clotted, offensive; flow only when moving about. Sensation as though all organs would escape, especially uterus ${ }^{[18]}$.

Millefolium: Menses-too profuse and of too long duration. Barrenness from profuse menstruation. Suppressed menses with epileptic attacks, pain in the stomach and cough with bloody expectoration. Vicarious menstruation. Uterine haemorrhage after great exertions. Haemorrhages from various organs, brought on by over-exertion.

Natrum Muriaticum: Retention of the otherwise regular menses. Menses too profuse and attended with colic, the discharge being black, flowing day and night. Melancholy mood. Sad and dejected mood, aggrarated by consolation from friends. Terrible sadness during menstruation. Sterility, with too early and too profuse menstruation (Calc. c.). Prolapsus uteri with aching in the lumbar region, better when lying on the back. Aversion to sexual intercourse, which is painful from dryness of the vagina. Natrum muriaticum is especially applicable to those cases of uterine diseases that have been subjected to topical applications of Nitrate of Silver, and also to complaints of old women ${ }^{[16]}$.

Jahr says: "Primary effect, shortening of the menses; secondary effect, extension." Menstrual blood dark and flowing day and night.

Nux Moschata: Irregular both in regard to time and quantity. Menses too late, sometimes too early. The discharge is dark and thick. Menorrhagia, blood thick and dark in women subject to irregular menstruation. Menses scanty or suppressed, from fright, from over exertion, from cold or from debility. Irritability about the pelvic viscera at each menstrual period, the uterus and ovaries are much swollen and sensitive to pressure. Nausea and vomiting from uterine irritation. Uterine displacements ac companied with intolerable drowsiness, faintness, dryness of the tongue, mouth and throat, distention of the abdomen, and outward pressure in the small of the back. Prolpsus uteri et. vagina. Hysterical complaints. Globus hystericus.

Pulsatilla Nigricans: Retarded or delayed menses in young girls at the age of puberty or in women at the menopause, accompanied with cramp-colic, chilliness, nausea, vomiting, trembling of the feet and complaints of various kinds. Uterine cramps, like labor-pains. Contractive pain in the left side of the uterus, obliging her to bend double. Metrorrhagia, at times very profuse, at others intermittent and mixed with clots. Post partum, secondary haemorrhage, from retained placenta or coagula. Threatened abortion, when the flow is intermittent. Leucorrhoea in women subject to uterine irregularities, anaemic patients, or in those of venous constitutions. Prolapsus-uteri with pressure in the abdomen and small of the back, as from a stone, the limbs tend to go to sleep and there is much ineffectual urging to stool. Indurations in the vagina.

Trillium Pendulum: Uterine Haemorrhage with sensation as though hips and back were falling apart ${ }^{[17]}$. Haemorrhage from fibroids. Prolapse with great bearing-down. Premature 
and profuse. Gushing of bright red blood from the uterus on the least movement. Profuse flow of dark, thick and clotted blood, continuing at intervals for several days. Profuse flow during the climacteric period, discharge dark, thick or clotted blood ${ }^{[16]}$.

Sabina: Too early and too profuse. Menstruation continues too long. Debilitating menses with abdominal spasms. ${ }^{16}$ Metrorrhagia is increased by the least motion, but sometimes better from walking. Uterine haemorrhage at the menstrual period, after abortion or parturition, attended with drawing, cutting, pressing pains, from the small of the back or more particularly, from the last lumbar vertebra through the pubis and down into the thigh. The blood is bright red or dark, also in clots, sometimes alternating.

Secale Cornuatum: Atonic haemorrhage during the critical period $^{16}$. Haemorrhage from atony of the uterus, especially after protracted and exhausting labor. Abortion attended with copious flow of dark, liquid blood, mingled with foulsmelling coagula, which is increased by the slightest motion. Passive haemorrhage of disorganized blood. Suppression of the menses in thin, scrawny, married women, who suffer a great deal at each menstrual epoch with continued, long-lasting, forcing pains in the uterus.

Thlaspi Bursa Pastoris: Haemorrhagic and uric acid diathesis. Menses bright red and profuse, too frequent, copious. Every alternate period very profuse. Sore pain in uterus on rising. Uterine bleeding with violent uterine colic. Uterine fibroids with cramps and expulsion of clots ${ }^{[23]}$.

Ustilago: Uterus hypertrophied. Vicarious menstruation. Profuse menses after miscarriage. Discharge of blood from slightest provocation [17]. Post-partum Haemorrhage. Persistent oozing of a dark, mahogany-colored, semi-fluid blood slightly coagulable, though occasionally forming long, black, stringy clots similar to those indicating Crocus. Irregular menstruation. Scanty menstruation with ovarian irritation. Ustilago very much resembles Secale in its general physiological action: both control uterine haemorrhage and promote uterine contraction in a similar [16].

Xanthoxylum: Painful haemorrhages, after pains; Neuralgic Dysmenorrhoea. Pain in back and down legs. Sluggish capillary circulation. Ovarian neuralgia with pain in loins and lower abdomen; worse left side. Leucorrhoea with amenorrhoea ${ }^{[19]}$.

- A recent study with a double blind randomized clinical trial showed the efficacy of remedy Ipecacuanha (Ipecac) in reducing pain, enhancing the quality of life of women with high bleeding and ultimately in reducing AUB. They have shown the effectiveness of the homoeopathuc remedies in Abnormal uterine bleeding ${ }^{[24]}$.

- Another study depicted the efficacy of Sabina, Cimicifuga and Thlaspi bursa in management of DUB while stating Sabina was more useful wherein the flow was bright red in colour, profuse, prolonged with pain radiating from back to the front of abdomen, flow on least exertion while cimicifuga in cases of DUB with dysmenorrhoea wherein the pain radiated from the back down the thighs, bleeding profuse dark coagulated.
Thalspi bursa was observed to work well when the flow is dark, gushing with clots and with reduced gap between periods ${ }^{[25]}$.

- From many researches and case studies done earlier, thorough case taking, prescribing on the basis of totality keeping in view of fundamental laws, a Homoeopath can derive excellent results and can serve mankind. The law of similar will direct to curative remedies for all that are curable and comfort such as are incurable, if we can keep our selfish ends in subjection ${ }^{[26]}$.

- Another study depicted that Arnica and Bellis per helped in reducing postpartum haemorrhage. After major blood loss after delivery as compared to placebo group, $\mathrm{Hb}$ levels remained same while the placebo group showed marked decrease in Hb levels ${ }^{[28]}$.

\section{Conclusion}

Menstruation is an important indicator of woman's health. Menstruation is affected by many factors including gynaecological diseases, mental stress, nutritional status and other environmental factors. Early diagnosis and standardized treatment are beneficial to long term reproductive health. Complications like severe anaemia can be prevented and hemodynamic stability is attained. Homoeopathy through its holistic approach, individualized treatment aims at management of abnormal bleeding patterns in order to achieve improved quality of life.

\section{References}

1. Dutta DC, MBBS, DGO, MO. D C Dutta's Textbook of Gynecology, Revised and reprint of Sixth edition 2013, 195.

2. Tortora GJ, Derrickson BH. Principles of anatomy and physiology. John Wiley \& Sons 2018.

3. Hall JE. Guyton and Hall textbook of medical physiology e-Book. Elsevier Health Sciences 2010.

4. Jonathan S Berek. MD, MMS. Berek \& Novak's Gynecology. Lippincott Williams \& Wilkins; $14^{\text {th }}$ Edition, First Indian Reprint 2007, 172-173.

5. Faruqui AA. Abnormal Uterine Bleeding: A Doctor Centric Survey on Prevalence, Management and Limitations in Indian Context. Obstetrics and Gynecology Research 2019;2:059-66.

6. Suseela TL, Parveen S, Archana D, Prasanna KS, Harini N, Pravallika V. A Study on Incidence, Clinical Profile and Prescribing Pattern in Abnormal Uterine Bleeding In a Tertiary Care Teaching Hospital. International Journal of Research and Review 2019;6(11):548-60.

7. Mishra D, Sultan S. FIGO's PALM-COEIN classification of abnormal uterine bleeding: A clinicohistopathological correlation in Indian setting. J Obstet Gynaecol India 2017;67:119-25.

8. Asma Ansari, Uzma Urooj. Study of causes behind abnormal uterine bleeding according to PALM-COEIN classification at a tertiary care hospital; J Park Med Assoc 2020;(1):154-155.

9. Lucy whitaker, Hilary OD. Critchley. Abnormal uterine bleeding; Best Pract Res Clin Obstet Gynaecol 2016; 34:54-65.

10. Munro MG, Critchley HOD, Fraser IS. FIGO Menstrual Disorders Committee. The two FIGO systems for normal and abnormal uterine bleeding symptoms and classification of causes of abnormal uterine bleeding in 
the reproductive years: 2018 revisions. Int J Gynaecol Obstet. 2018;143(3):393-408.

11. Hasan RA, Zia A. Heavy Menstrual Bleeding and Bleeding Disorders in Adolescents: A Primer for the Primary Care Physician. In: Kamat D., Frei-Jones M. (eds) Benign Hematologic Disorders in Children. Springer, Cham 2021

12. World Health Organization multicenter study on menstrual, ovulatory patterns in adolescent girls. II. Longitudinal study of menstrual patterns in the early postmenarcheal period, duration of bleeding episodes and menstrual cycles. World Health Organization Task Force on Adolescent Reproductive Health. J Adolesc Health Care 1986;7:236-44.

13. Copperthwaite A. n.d. Disorders of Menstruation. New Delhi: B Jain Publishers.

14. Kent JT. What the doctor needs to know in order to make a successful prescription. Sett Dey 1957.

15. Roberts HA. The Principles and Art of Cure by Homoeopathy: A Modern Textbook. B. Jain Publishers 1997.

16. Henry Minton. Uterine Therapeutics; New York: A. L. Chatterton publishing Co, 1884.

17. William Boericke. Pocket manual of Homoeopathic material medica and repertory; Comprising of The characteristic and guiding symptoms of all remedies including Indian drugs., B.Jain Publishers Pvt. Ltd.

18. Robin Murphy. Lotus Materia Medica, Third edition. B.Jain publishers.P.Ltd 2018.

19. Hallberg L, Hogdahl AM, Nilsson L, Rybo G. Menstrual blood loss: a population study, Acta Obstet Gynecol Scand 1966;45:320-351.

20. Allen JH. The chronic miasms. B. Jain Publishers; 1998.

21. Davis E, Sparzak PB. Abnormal Uterine Bleeding. [Updated 2021 Feb 10]. In: StatPearls [Internet]. Treasure Island (FL): StatPearls Publishing 2021.

22. Walker MH, Coffey W, Borger J. Menorrhagia. 2020 Sep 4. In: StatPearls [Internet]. Treasure Island (FL): StatPearls Publishing 2021. PMID: 30725595.

23. Robin Murphy, Lotus Materia Medica, B.Jain Publishers p. ltd, Third edition 2018.

24. Zohreh Naderi, Zarin Dadkhah, Ali Karimi, Razieh Hashemi, Mayam Aghaee, Abbas Talallaii et al. Effectiveness of Homoeopathic Treatment for Abnormal Uterine Bleeding: A double blind randomized clinical trial, Hosp Practices and research. 2020;5(3):92-97.

25. Parth Aphale, Atul Rajgurav. To study the efficacy of Sabina, cimicifuga and thlaspi bursa in management of cases of Dysfunctional Uterine Bleeding, International journal of reproduction, contraception, Obstetrics and Gynaecology Aphale P et. Al. Int J Reprod Contracept Obstet Gynecol 2017;6(1):133-140.

26. Kent JT. NEW REMEDIES Clinical cases lesser writings aphorisms and percepts, B Jain publishers P. Ltd., reprint edition. 2004

27. Khafaga A, Goldstein SR. Abnormal Uterine Bleeding. Obstet Gynecol Clin North Am. 2019;46(4):595-605. doi: 10.1016/j.ogc.2019.07.001. Epub 2019 Sep 25. PMID: 31677744.

28. Oberbaum M, Galoyan N, Lerner-Geva L, Singer SR, Grisaru S, Shashar D et al. The effect of the homeopathic remedies Arnica montana and Bellis perennis on mild postpartum bleeding--a randomized, double-blind, placebo-controlled study--preliminary results. Complement Ther Med. 2005;13(2):87-90. doi: 10.1016/j.ctim.2005.03.006. PMID: 16036165. 